\title{
ESTRATEGIAS FAMILIARES DE LOS JÓVENES MARROQUÍES EMIGRADOS A ANDALUCÍA (ESPAÑA) EN LOS ÚLTIMOS AÑOS
}

\author{
Alberto Capote*
}

\begin{abstract}
Durante la pasada década los marroquíes han constituido la nacionalidad extranjera extra-comunitaria más numerosa en la llegada de inmigrantes a España. Se ha tratado eminentemente de una población joven bastante heterogénea procedente tanto del medio rural como urbano y relativamente bien formada. Sus motivaciones para emigrar revelan un descontento juvenil bastante generalizado, aunque de distinta naturaleza según los perfiles sociales. En este artículo analizamos el papel de la familia a la hora de tomar la decisión de emigrar al extranjero: a cuántos miembros de la familia concierne dicha experiencia y qué estrategias se han puesto en marcha. Los resultados de nuestro estudio nos revelan hasta que punto la familia juega un papel esencial en los procesos de partida de los jóvenes marroquíes emigrados a España. Ahora bien, más que de una decisión adoptada en el seno familiar se trata, para muchos de ellos, de una cadena de ayuda entre hermanos que comparten un mismo descontento generacional. Este artículo indaga pues en las primeras etapas de los proyectos migratorios y muestra además algunos cambios que han tenido lugar en la juventud marroquí en los últimos años.
\end{abstract}

Palabras clave: inmigración, familia, Marruecos, España, descontento juvenil.

\section{Introducción}

Las razones que suelen ser invocadas en la literatura especializada para explicar la significativa afluencia de inmigrantes marroquíes a España durante la pasada década son la proximidad geográfica entre los dos países, la percepción de que la entrada era más accesible para los migrantes

\footnotetext{
* Departamento de Geografía Humana, Universidad de Granada. Granada/España.
} 
clandestinos en relación con otros países europeos, el fuerte crecimiento económico en España hasta el inicio de la crisis en 2008 y la demanda de mano obra en determinados sectores productivos durante este periodo, particularmente la agricultura intensiva de tipo industrial y el fenómeno, sin precedentes, del boom del sector inmobiliario español ${ }^{1}$. Conexo a estos factores no podemos olvidar el desarrollo de las redes sociales migratorias, las cuales juegan un papel fundamental, principalmente, en la elección de los destinos y en el proceso de tomar la decisión de emigrar al extranjero.

La función de las redes migratorias, entendidas como "el conjunto de relaciones interpersonales que vinculan a los inmigrantes, los futuros migrantes y los no emigrados con los espacios de origen y de destino, a través de nexos familiares, de amistad y de un origen comunitario compartido" ${ }^{2}$, ha constituido una de las líneas de investigación más fecundas en los últimos años en los estudios sobre la inmigración internacional. Isidro Maya ${ }^{3}$ sintetiza en tres las funciones de estas redes: por una parte, su cometido a la hora de tomar la decisión de emigrar, en el sentido de que es más fácil decidirse cuando se dispone de vínculos, ya sean familiares, de paisanaje, de amistad o de otro tipo, en los posibles contextos de recepción; en segundo lugar, contribuyen a extender el abanico de los candidatos potenciales a la emigración: en un principio, los mecanismos de selección apuntan a unos perfiles determinados entre los pioneros (jóvenes, varones, con actitud "emprendedora", en lo que a los marroquíes se refiere, por ejemplo), y posteriormente éstos ejercen su influencia para movilizar a personas con características y en situaciones diferentes; en último lugar, cabe destacar que facilitan el proceso de adaptación en los lugares de acogida, ya que la presencia de connacionales (familiares y amigos) contribuye a amortiguar las dificultades inherentes a las primeras fases de asentamiento. De este modo, los primeros lugares de instalación se van regenerando como espacios de inmigración a través de las cadenas familiares o de otra índole ${ }^{4}$.

La familia ocupa un lugar primordial en estos estudios. A menudo se adopta la perspectiva de que los movimientos migratorios obedecen con más

1 LAZAAR, Mohamed. L'immigration marocaine en Espagne, p. 83-93; CACHÓN, Lorenzo. La España inmigrante: marco discriminatorio, mercado de trabajo y políticas de integración.

MASSEY, Douglas S. The ethnosurvey in theory and practice, p. 1498-1522

3 MAYA, Isidro. La formación de comunidades de inmigrantes: desplazamiento en cadena y contexto de recepción, p. 83-91.

4 MASSEY, Douglas S.; GOLDRING, Luin; DURAND, Jorge. Continuities in transnational migration: an analysis of nineteen mexican communities, p. 1492-1533; GOZDIAK, Elzbieta e BUMP, Micah. Poultry, Apples, and New Immigrants in the Rural Communities of the Shenandoah Valley: An Ethnographic Case Study, p. 149-164. 
frecuencia a una decisión familiar que a una opción de carácter meramente individual: es el caso, por ejemplo, de aquellas familias que en contextos de crisis se organizan para enviar a uno de sus miembros a otro lugar con el fin de asegurarse una renta migratoria ${ }^{5}$. De otro lado, hay estudios que ponen de manifiesto que las motivaciones para emigrar varían sustancialmente en función de la etapa vital en la que se encuentran los futuros inmigrantes: así, las inquietudes que persiguen un interés más individualizado suelen ser más frecuentes entre los jóvenes que entre personas de más edad donde predominan más intereses de grupo de tipo familiar ${ }^{6}$. A modo de ejemplo, dentro de esta última línea distintos autores mexicanos sitúan la emigración de los estados limítrofes de Tamaulipas y Veracruz hacia los Estados Unidos: la decisión de emigrar es básicamente individual, aunque ésta pueda estar fuertemente influenciada o condicionada por otras experiencias familiares $u$ otras personas del círculo más próximo que han emigrado con anterioridad ${ }^{7}$.

Según Pedone ${ }^{8}$, en el estudio de las redes migratorias familiares es fundamental tomar en consideración las pautas culturales propias del colectivo que se esté estudiando. En lo que a la población marroquí se refiere tenemos que tener presente el papel esencial de la institución familiar en la sociedad, así como los cambios acaecidos en los últimos años en ella. Existe unanimidad en considerar que una de las variaciones demográficas más importantes en el Magreb en los últimos veinte años ha sido el retraso en la edad del primer matrimonio ${ }^{9}$. Los cambios económicos, sociales y culturales que han tenido lugar en las sociedades magrebíes han introducido algunas modificaciones en las prácticas matrimoniales y en la institución familiar en general. La escolaridad se ha ido generalizando en Marruecos cada vez más. A la par de esta mejora en los indicadores de educación, nos encontramos con la irrupción de nuevos descontentos entre los jóvenes. El desempleo en Marruecos afecta particularmente a las personas mejor instruidas. No hay que olvidar tampoco el bajo nivel de salarios, así como el mayor acceso de

5 BERRIANE, Mohamed. Los focos migratorios marroquíes y la emigración hacia España, p. 128130; GONIN, Patrick, KOTLOK, Nathalie e LIMA, Stephanie. Entre réseaux et territoires, des mobilisations multiséculaires pour le développement. Réseaux migratoires et communes rurales dans la région de Kayes, Mali, p. 265-278.

6 KLEY, Stefanie. Explaining migration as a process of cumulative causation in the life of course.

7 PÉREZ MONTEROSAS, Mario. Las redes sociales en la migración emergente de Veracruz a los Estados Unidos, p. 136-169; IZCARA PALACIOS, Simón Pedro. Redes migratorias versus demanda laboral: los elementos que moldean los procesos migratorios, p. 39-59.

8 PEDONE, Claudia. Diversificación de las cadenas migratorias ecuatorianas hacia el mercado de trabajo agrícola de Murcia, España, p. 255-271.

9 FARGUES, Philippe. La femme dans les pays arabes: vers une remise en cause du système patriarcal?, p. 1-4; EL HARRAS, Mokhtar. Marruecos: la diversificación de las estructuras y de las relaciones familiares, p. 34-36. 
la juventud a la información y la toma de contacto con otras realidades a través, principalmente, de las nuevas tecnologías. Asimismo, como sostiene Rachik $^{10}$, los jóvenes del medio rural y los del medio urbano en la sociedad marroquí actual comparten una característica común: la de desmarcarse en diferentes aspectos de la vida social de la generación de sus progenitores. En efecto, las relaciones intergeneracionales en Marruecos en el seno de la familia se encuentran en un proceso de mutación en el que los jóvenes buscan una mayor autonomía en relación con sus ascendentes y un cambio de valores ${ }^{11}$. A ello ha contribuido, entre otras razones, el incremento de la distancia entre el nivel de estudios de los progenitores y el de sus hijos.

En este artículo analizamos la participación de la familia en los procesos migratorios de partida de los jóvenes marroquíes en Andalucía (sur de España) durante la pasada década a partir de un estudio empírico realizado en varios municipios andaluces. Las cuestiones concretas que abordamos son las siguientes: en qué medida la emigración está presente en las familias de estos jóvenes marroquíes; qué tipo de estrategias familiares se han puesto en marcha en los procesos de partida; y por último, consideramos que es también muy interesante observar si en dichos procesos se aprecian los cambios sociales sobre la juventud marroquí que se han descrito anteriormente, los cuales se traducen en un descontento bastante generalizado tanto en el medio rural como urbano y que afectan a una juventud muy heterogénea que, bastante a menudo, se engloba en un todo homogéneo.

\section{Metodología de la investigación}

La metodología de nuestro estudio ha integrado tanto técnicas cuantitativas como cualitativas de recogida y análisis de la información. Por una parte, la realización de una encuesta en cinco municipios andaluces receptores de inmigrantes marroquíes. La muestra la han compuesto 213 personas de nacionalidad marroquí (el 66\% hombres) ${ }^{12}$. Es importante subrayar que nuestro objetivo no ha sido alcanzar estadísticas de ponderación con los resultados obtenidos, sino obtener una serie de indicadores de carácter cuantitativo para completarlos con la información cualitativa. De este modo, se realizaron igualmente 33 entrevistas semi-estructuradas a una selección de los inmigrantes encuestados y algunos allegados de

\footnotetext{
${ }^{10}$ RACHICK, Hassan. Jeunesse et changement social, p. 191-216.

11 KARAM, Azza. Los jóvenes en el Mediterráneo: retos y oportunidades culturales de cambio, p. 193-200.

12 La muestra ha sido elaborada mediante un muestreo sistemático con arranque aleatorio, previa ordenación de los efectivos por sexo y edad a partir del Padrón de habitantes (registro anual de la población en los ayuntamientos).
} 
éstos, quienes han sido, generalmente, familiares. El trabajo de campo se realizó en cinco municipios de dos provincias andaluzas. En la provincia de Granada en dos con predominio de la agricultura intensiva de carácter industrial (Albuñol y Zafarraya) y, como contrapunto urbano, en la capital granadina, ciudad eminentemente de servicios con gran actividad ligada al turismo; en la provincia de Córdoba, en dos municipios de interior (Lucena y Aguilar de la Frontera), a los que los marroquíes han llegado a partir de una migración interna en España y también se emplean en la agricultura. Nuestro propósito ha sido pues abarcar una gama variada de los contextos locales de la inmigración en Andalucía.

Según se deduce de nuestro estudio, los marroquíes emigrados a España han sido, sobre todo, jóvenes concentrados en las primeras edades laboralmente activas. En consonancia con esto la decisión de emigrar se tomó a una edad muy temprana, siendo incluso menores de edad en casos bastante numerosos, lo que explica que en su mayor parte los encuestados llegaran a España antes de contraer matrimonio. Sin embargo, una parte bastante destacable de ellos se ha casado en un intervalo de tiempo relativamente corto. Esto permite pensar que el matrimonio ha sido uno de los fines asociados a los proyectos migratorios de un sector de estos jóvenes, siquiera colateralmente. Cabe destacar que el perfil de la inmigración marroquí en la ciudad de Granada es bastante diverso en comparación al contexto andaluz general: predominan las personas de origen urbano y con mejores niveles de formación. De hecho, la capital granadina es conocida por recibir el mayor número de estudiantes marroquíes, muchos de los cuales cambian con el tiempo de proyecto migratorio y se insertan con desigual fortuna en el mercado de trabajo. Por último, es importante señalar la presencia femenina con proyectos migratorios que no se ajustan al esquema clásico de la reagrupación familiar. Aquí estamos hablando principalmente de mujeres de origen urbano que han emigrado de manera casi autónoma.

\section{Trayectoria migratoria familiar de los inmigrados marroquíes en Andalucía}

Una abrumadora mayoría de los encuestados (72\%, 153 de 213) afirmó que al menos un miembro de su familia cercana contaba también con una experiencia migratoria en el extranjero, independientemente de si se produjo o no posteriormente un retorno definitivo a Marruecos. La encuesta pone además de manifiesto una alta frecuencia de las migraciones de hermanos $(69,4 \%)$ y una constancia de padres inmigrados mucho menos frecuente (15\%, la diferencia entre los padres y las madres es insignificante). 
Por tanto, la primera observación a poner de relieve es la existencia de una componente bastante destacada que se caracteriza como una migración de jóvenes colaterales. La segunda observación en la que ponemos el acento es la alta concentración geográfica de la experiencia migratoria de estos familiares en España: éste ha sido el destino (o uno de ellos) para el 82\% de los hermanos emigrantes, el 90,3\% de las madres y el 53,1\% de los padres. Es más, el 72,3\% afirma igualmente tener a un familiar lejano (tíos, primos) que también emigró a España. La lectura de estos resultados nos lleva a afirmar que se trata de una emigración en buena medida desconectada de filiares migratorias anteriores con destino a otros países europeos (fundamentalmente Francia y Bélgica). Dicho de otro modo, tal parece que los contextos más recientes de la emigración marroquí se han traducido en una selección de los candidatos hasta cierto punto específica y "novedosa" con respecto a etapas precedentes. A ello no es ajeno que entre los orígenes geográficos nos encontremos con áreas de reciente incorporación en los flujos migratorios al extranjero, como son las provincias interiores (Beni Mellal, Juribga, El Keláa), en contraposición con otras con una mayor trayectoria como puede ser el caso de Nador. Pese a la clara concentración de los familiares en España, cabe destacar la variedad de los destinos, particularmente en lo que se refiere a los padres, lo que evidencia el grado de dispersión de la emigración marroquí en comparación con los otros dos países del Magreb central, Túnez y sobre todo Argelia, cuyos destinos son mucho más concentrados.

Profundizaremos más en la geografía de los destinos de los hermanos emigrados de las personas encuestadas. En el mapa 1 hemos indicado sus provincias de residencia en España. Si bien apreciamos cierta dispersión (sobre todo por la comunidad andaluza, Madrid y Barcelona), lo que más sobresale es la concentración en las provincias de Córdoba y Granada, es decir, las provincias en las que se ubican los municipios en los que se ha aplicado el cuestionario. De hecho, el 42\% de los encuestados afirmó tener al menos a un hermano viviendo en su mismo municipio de residencia, proporción aún más alta en Albuñol (58\%), donde se localiza también la frecuencia relativa más alta de otros familiares que también viven en España (83\%).

El porcentaje de respuestas positivas es mayor en las mujeres $(79,4 \%)$ que en los hombres $(67,8 \%)$. Datos que nos revelan que los proyectos migratorios en el sexo femenino se insertan con más frecuencia en el marco familiar, y no se limitan siempre a la reagrupación por parte del cónyuge. Aunque no han sido muy numerosos, durante el trabajo de campo hemos conocido casos en los que fue la hermana de un encuestado quien inició 
la cadena migratoria familiar. La conclusión que podemos extraer de la lectura de estos resultados es que el efecto de arrastre de los colaterales no se desencadena siempre a partir de un hombre, sino que adopta múltiples formas en las que las mujeres a veces adquieren un papel destacado aunque no de la misma forma que sucede, por ejemplo, con la inmigración femenina procedente de América Latina.

\section{MAPA 1. Provincias españolas a las que emigraron los hermanos de los encuestados}

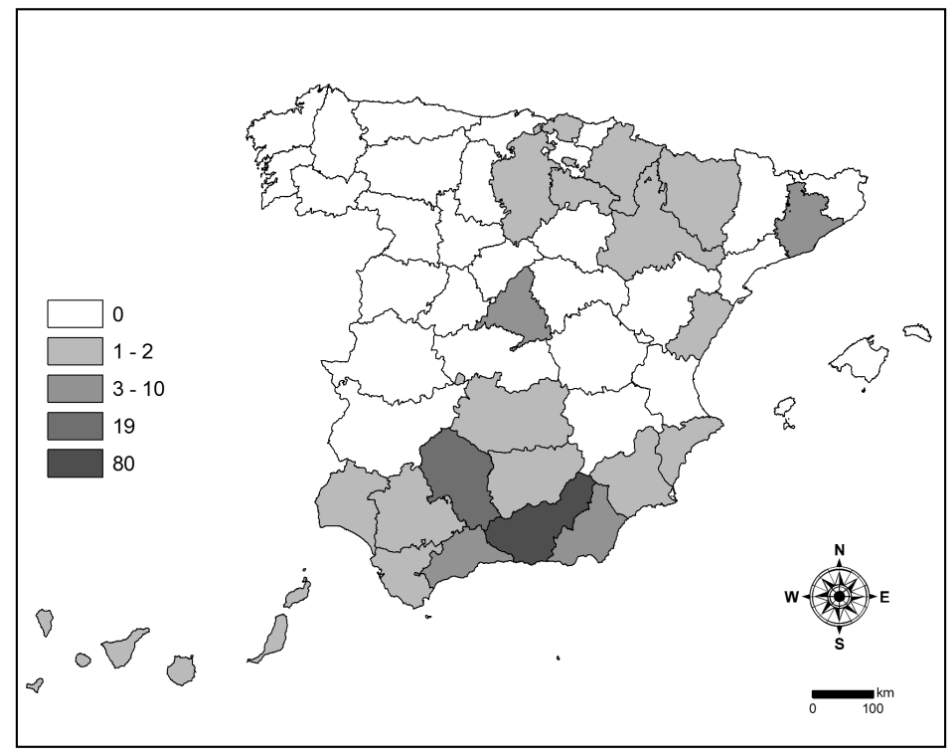

Fuente: elaboración propia.

Son también llamativos los resultados en función de las áreas de procedencia, sobre todo porque la región Rif y Oriental (norte de Marruecos) aventaja claramente a las otras regiones migratorias en las respuestas positivas (83\%, frente al $66,6 \%$ del eje atlántico en torno a Casablanca y Rabat, el porcentaje más bajo). Una cultura de la movilidad muy arraigada en la región y las relaciones históricas, culturales y lingüísticas con España, así como la proximidad geográfica ${ }^{13}$, justificarían esta mayor proporción de familiares emigrados particularmente en lo que se refiere a las provincias de Nador y Alhucemas. De manera específica, tenemos que tener en cuenta los vínculos establecidos a través de los estudios. Como se ha dicho anteriormente, la ciudad de Granada es el destino privilegiado para los estudiantes marroquíes, sobre todo para aquellos que lo hacen

$\overline{{ }^{13} \text { BERRIANE, op. cit. }}$ 
desde un centro de enseñanza español ubicado en Marruecos. Se observa a partir de los datos recogidos en el trabajo de campo que es una práctica que comparten con asiduidad varios hermanos de las provincias de Nador y de Alhucemas:

Sí, porque mis padres nos han matriculado a mis tres hermanas y luego mis tías que también habían estudiado en el colegio éste, un colegio-instituto y todas mis tías, mis hermanos, mis primas, bueno, todas [...]. ¿Por qué?, pues... es que mis abuelos también hablaban español, conocían mucho la cultura española y eso, cuando era la época del protectorado... Sí, entonces, habían convivido con españoles y yo que sé y entonces, creo que venía de eso. Aparte que yo creo también porque buscaban un colegio bueno, buena educación para que el día de mañana cuando acabes, puedas irte a estudiar a una buena facultad, isabes? Pues yo creo que eso era de nuestros antepasados (GR_10).

Es también interesante conocer a cuántos hermanos concierne la experiencia migratoria en el extranjero. La media para la suma de los cinco municipios es de 2,1 (con un coeficiente de variación del 70\%). Para cerca de la mitad de los encuestados se trata de dos hermanos como mínimo e incluso hay un $18 \%$ en el que todos han emigrado (gráfico 1). Así, cabe afirmar que una vez que un miembro de la familia emprende el proyecto migratorio es bastante probable que se produzca un efecto en cadena. En el cuestionario preguntamos a los encuestados cuándo habían emigrado sus familiares: si lo habían hecho antes que los encuestados mismos, al mismo tiempo o después. Como en un principio cabía esperar, la emigración de los progenitores tuvo lugar la mayoría de las veces con anterioridad (gráfico 2); solamente para un grupo reducido la salida ocurrió después (entre los cuales se encuentran algunos padres de antiguos estudiantes en Granada, ciudad en la que pasan largas temporadas al año). Las madres, en cambio, lo hicieron con menos frecuencia antes que sus hijos. Este dato se explica porque ha sido más frecuente reagrupar a las madres, sobre todo cuando éstas enviudan y la mayor parte de los hijos (si no son todos) se encuentran en el extranjero. Por último, en lo que respecta a los hermanos emigrados, las proporciones se encuentran más repartidas, lo que incide aún más en el efecto en cadena de los movimientos migratorios entre colaterales. A este respecto, es interesante hacer notar que un tercio de los encuestados cuenta al mismo tiempo con un hermano que emigró antes que él mismo y otro que lo hizo después. 
GRÁFICO 1 - Hermanos con una experiencia migratoria internacional (\%)

a) Número de hermanos con una experiencia migratoria internacional

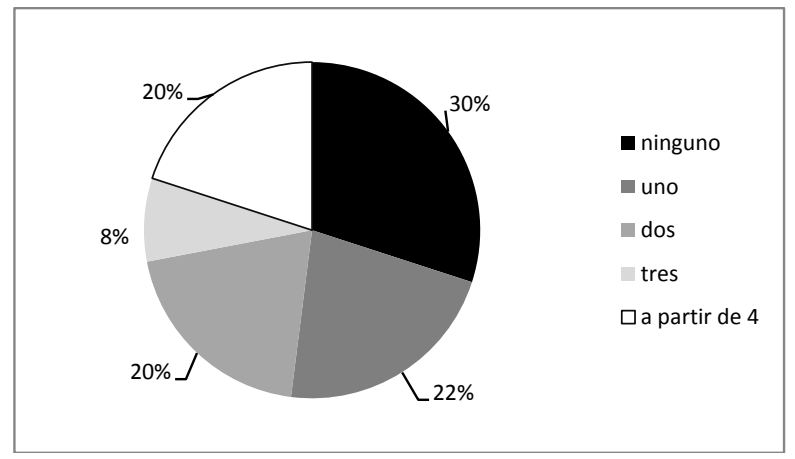

b) Porcentaje de hermanos con una experiencia migratoria internacional

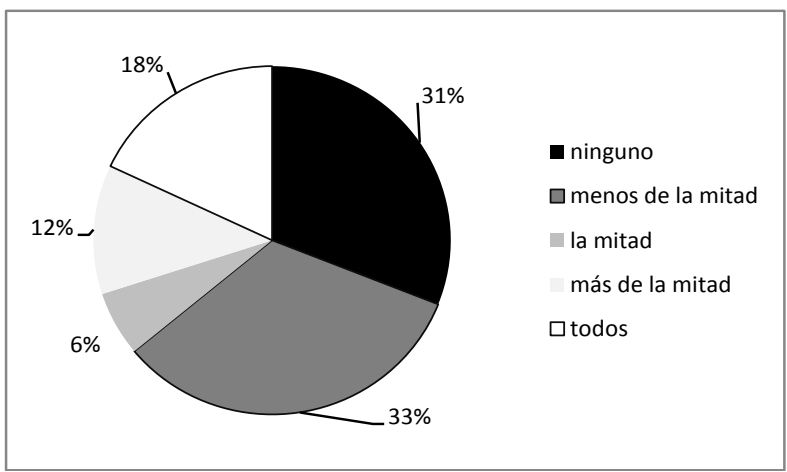

Fuente: elaboración propia.

GRÁFICO 2. Momentos en los que han emigrado los familiares de los encuestados en relación a ellos mismos (\%)

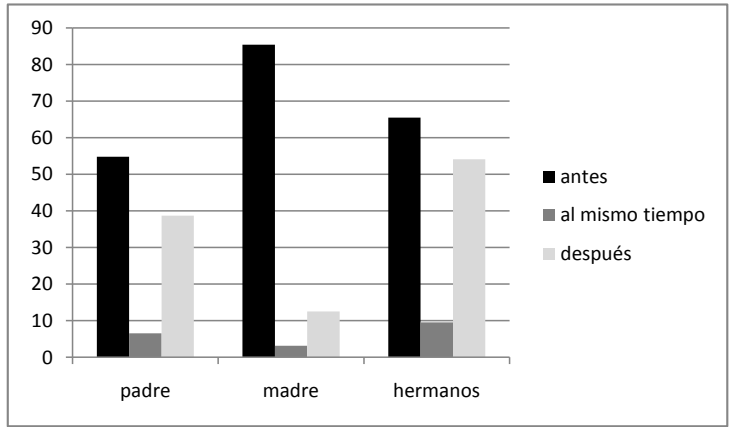

Fuente: elaboración propia. 


\section{La financiación del proyecto migratorio de partida y la participación de la familia}

Preguntamos también nuestros interlocutores en qué condiciones tuvo lugar la primera salida fuera de Marruecos: el medio de transporte utilizado y los gastos asociados a este primer viaje. En un principio cabe preguntarse qué interés pueden tener estas cuestiones para los objetivos de nuestra investigación. Sin embargo, qué duda cabe de que se trata de una experiencia trascendental en la vida de estas personas, máxime cuando esta salida se realizó utilizando algún medio clandestino, por los riesgos que con frecuencia conlleva: "Allí empiezan muchísimas preguntas, pero uno está salvado, está con la alegría de no haber muerto en el mar ni nada de eso, estaba uno contento..." (ZA_01), esta es la respuesta de un joven entrevistado en Zafarraya a la pregunta de cuáles fueron sus impresiones al llegar a su primer destino español. Además, nos proporciona algunas informaciones de interés: las condiciones dispares en las que se inicia la aventura migratoria y un primer indicador relativo al grado de participación de la familia en el proyecto inicial. Una amplia mayoría de los encuestados realizó el primer viaje en ferry (74\%, 158 de 213). El medio de transporte menos utilizado fue el avión (sólo un 5,1\%), lo que se explica fundamentalmente por la proximidad geográfica entre los dos países. El resto, sin contar con las personas que no respondieron a esta pregunta, lo hizo por un medio clandestino $(18,7 \%$, generalmente en patera).

Evidentemente, emprender el proyecto migratorio conlleva un coste, además de psicológico, económico. Éste puede llegar a ser especialmente alto cuando se recurre a algún medio clandestino. El grado de juventud de la muestra nos hacía presagiar una participación esencial por parte de la familia en los gastos iniciales. Esta cuestión nos puede dar algunas pistas en torno a si el proyecto migratorio se inició de manera autónoma o en concertación con terceras personas. Como se ha dicho anteriormente, desde diversos enfoques de las redes sociales se sostiene que generalmente la decisión de emigrar se organiza en el seno familiar y no responde a una iniciativa estrictamente individual.

En el cuestionario se incluían cuatro posibles respuestas sobre el coste del viaje inicial, de las cuales se podían seleccionar como mucho dos: a) los gastos corrieron por parte del mismo encuestado; b) de la familia en Marruecos; c) de los familiares emigrados; d) y una cuarta categoría para incluir otro tipo de situación. En raras ocasiones ha habido un solapamiento en las respuestas. El 41\% afirmaron que ellos mismos financiaron los gastos iniciales. Si descontamos de este porcentaje aquellos casos en los que también 
se aludió a algún miembro de la familia, la proporción desciende a un 37,5\%. Para una proporción notable $(39,4 \%)$, el coste fue financiado por la familia en Marruecos. En cambio, la contribución de la familia emigrada fue más modesta $(13,1 \%)$. Si bien no tiene por qué ocurrir siempre, es muy probable que si un joven se desplaza para estudiar a otro país, los padres contribuyan sustancialmente en los gastos, particularmente cuando se inician los estudios universitarios a los 18 años. Efectivamente, en Granada (donde un gran número de encuestado emigró con un visado de estudios) la participación de la familia en Marruecos supera el $50 \%$ de las respuestas. En cambio, las respuestas disminuyen sustancialmente entre los inmigrantes marroquíes que se encuentran en los otros cuatro municipios andaluces, los cuales se ajustan más al patrón más común del joven emigrado que encuentra trabajo en el medio agrícola y cuya primera motivación eminentemente económica.

No carece de interés examinar los resultados en función de las condiciones administrativas de partida. Como hemos evocado anteriormente, el coste económico de una migración utilizando algún medio clandestino puede llegar a ser especialmente elevado (patera, documentación falsificada...). Cabe pensar que en estos casos la participación familiar fue indispensable. En lo que se refiere a la reagrupación familiar, lo más lógico es que fuera la familia instalada en España la que interviniera. Los datos que arroja la encuesta confirman, con sus matices, estas premisas de partida. Ratificamos que para los que llegaron con un visado de estudios, los gastos fueron financiados por los familiares no emigrados, de ahí que esta categoría de respuesta sea muy relevante en la capital granadina (porcentaje). Efectivamente, para los casos de reagrupación familiar la ayuda vino de la familia emigrada (aquí se incluiría también el cónyuge). El autofinanciamiento es más frecuente entre los que emigraron con un visado de turista, un contrato en origen o clandestinamente. Sobre estos últimos, no se cumple la premisa de la que partíamos: si bien uno de cada tres contó con la participación familiar, lo que destaca es que un grupo importante asumieron por sí solos estos gastos. En algunos casos la contribución de la familia tuvo lugar una vez llegaron a España, de manera imprevista, cuando las "mafias" que gestionaron el viaje exigieron más dinero del que se había pactado. Estos jóvenes tuvieron entonces que recurrir a pedir ayuda a los familiares. Por otro lado, una parte organizó el viaje al margen de la familia, sin ni siquiera informar del día de la salida, a menudo con la intención de evitar en los padres el temor y la angustia que conllevaría el viaje: "Mi padre no sabía nada. Yo he cogido mi dinero, lo que he sudado y un poco más que me lo presta la gente allí y ninguno sabía que yo venía a España entre la vida 
y la muerte" (ZA_01). Así, se desprende de estos datos que muchos de estos jóvenes no pudieron dar el paso hasta reunir el presupuesto necesario.

Llegados a este punto, iqué valoración podemos hacer de la participación familiar a la hora de tomar la decisión de emigrar? Le Bras y Fargues ${ }^{14}$ apuntan que los jóvenes de los países del sur del Mediterráneo que emigran por razones laborales no sólo lo hacen para contribuir a la subsistencia de la familia, sino por ellos mismos, por construir sus propios proyectos, por los objetivos que se trazan en función de la formación académica, o por los anhelos que despiertan los medios de comunicación y el contacto con los paisanos emigrados. La exclusión no sólo se reduce al mercado laboral, sino que a su vez se manifiesta en otros ámbitos interrelacionados como el acceso al matrimonio y a una vivienda independiente de la de los padres. A este respecto, El Harras ${ }^{15}$ señala la tendencia cada vez mayor hacia la autonomía residencial por parte de las jóvenes parejas. La aspiración es combinar un espacio privado relativamente autónomo, preservando a su vez los vínculos directos con la familia parental.

No, que va, antes estaba estudiando, no pensaba en eso... después salí del colegio y me puse a trabajar con mi padre hasta el año 2000 [...]. Bueno, he pensado que era joven y en Marruecos sólo estaba en la casa, con mi padre, vivía con mi padre, trabajaba con él... me he dicho salgo y me busco la vida y ya está. Porque como tenía la familia aquí, he pensado me voy de aquí... por lo menos puedo buscar trabajo y tengo allí familia (GR_15).

Nunca he pensado en salir. Yo pensaba en trabajar, en buscar un futuro, en casarme y ya está. Pero yo no era el único, los otros amigos sólo me hablan de salir, me hablan del extranjero y son los que me han dado la idea de hacerlo (AG_01).

Cuando llegué a los 15 años pensé en dejar el aduar e irme a otro lado. Entonces mi padre me dijo que me tenía que quedar con mi hermano mayor, que estaba en Tetuán, allí tenía una tienda de ropa. Entonces me fui a Tetuán para ayudarlo y me quedé con él [...]. Después no me ha gustado estar allí. Mi hermano me ha dicho quédate conmigo un año y al otro te doy dinero para irte a España [...]. En Tetuán hace mucho frío... yo no tengo casi futuro... Si me quedo en Tetuán, no puedo comprarme un coche, no puedo comprar una casa, no puedo casarme... (LU_01).

\footnotetext{
${ }^{14}$ FARGUES, Philippe; LE BRAS, Hervé. Migrants et migrations dans le bassin de la Méditerranée.

${ }^{15}$ EL HARRAS, op. cit.
} 
No obstante, esto no nos no debe hacer perder de vista que muchos de estos jóvenes también contribuyen en la distancia, de manera dispar, a la economía de la familia en Marruecos. Como un inmigrado entrevistado en Zafarraya, oriundo de la provincia de Beni Mellal, en determinados casos que un hijo emigre "era un sueño para los padres, para los abuelos, para todos. Pero para los que no tienen dinero... De mi padre y de cualquier padre. De cualquier padre pobre que sueña con tener un futuro bueno. Que venga de parte de su hijo o de su hija o de quien sea" (ZA_01).

\section{Estrategias familiares entre hermanos en la inmigración marroquí a Andalucía}

Queda por indagar cómo se han ido enlazando las migraciones entre hermanos, qué estrategias se han desarrollado. Las situaciones son bastante diversas. Anteriormente hemos visto los casos de jóvenes que partieron con un visado de estudios después de haber cursado la secundaria en algún centro español (particularmente en las provincias marroquíes del norte). En este último epígrafe nos vamos a ceñir a algunos casos extraídos de las entrevistas semi-estructuradas que emigraron con la primera intención de trabajar. Comparten una característica común: los padres nunca emigraron o, si lo hicieron, fue con anterioridad y retornaron antes de que los entrevistados iniciasen por sí solos el proyecto migratorio.

Cabe empezar diciendo que no siempre los hermanos emigrados alientan a otro a que inicie el proyecto migratorio. En ocasiones lo que se intenta es justo o contrario: desmotivar o en todo caso retrasar la posible salida, a menudo porque no se dispone de las condiciones suficientes para ejercer como receptores de los recién llegados:

La primera vez me dijeron que era mejor que siguiera allí, que allí por lo menos estabas trabajando en lo mío, que tienes un trabajo tuyo, no te manda nadie, que en España la vida es difícil, pero no, yo le dije que me voy (GR_15).

... nadie estaba aquí, estaba nada más que Hassan (nombre ficticio del hermano). Y me dice quédate allí, quédate allí hasta que te busque un contrato o algo. Pero no me ha buscado nada. Por eso, porque era muy difícil buscar aquí en España un contrato. Nadie quiere dar un contrato (LU_01).

No obstante, esta actitud entra en contradicción durante los periodos vacacionales con la imagen de éxito que suele proyectar el emigrado. Sin duda, durante estos retornos temporales se tuvieron que gestar muchos de los futuros proyectos migratorios. Qué mayor estímulo para emigrar que 
disponer de alguien cercano con dicha experiencia. Lo que en un principio se podría concebir como inaccesible o muy lejano, se vuelve más factible, máxime cuando es el propio hermano quien lo ha conseguido. Por tanto, el desaliento o la espera que a veces se pretende exteriorizar no impiden que estos jóvenes se lancen por sí solos. Casi se podría decir que el efecto demostración se hace aún mayor o adquiere otras connotaciones de atracción.

Ahora bien, en la mayor parte de los testimonios la colaboración de los hermanos desde el momento inicial es muy manifiesta. Hemos comprobado que una forma bastante común de proceder ha sido que los hermanos emigrados a España negociasen en sus lugares de trabajo alguna oferta de trabajo, especialmente en los municipios españoles de agricultura intensiva. Pongamos algunos ejemplos ilustrativos. El primero se refiere a un joven entrevistado en Zafarraya, originario de Beni Mellal, que trabaja como temporero en la agricultura:

¿De mi familia, de los primos y eso? Hay un hermano, sí. Está aquí conmigo, lo he traído yo... Por la confianza he ganado el contrato. Porque si no fuera por la confianza, no me lo daría ninguno. Hombre, que él tenía tierra, tenía cortijo grande, tenía eso. Estuve trabajando con él, él me conoce y yo le conozco a él, por eso. Le digo me firmas a mi hermano o me voy. Un acuerdo, me firma a mi hermano aquí. Me interesa que mi hermano esté aquí. Si no me da, me voy a buscar otro. Que trabajo con él y me da eso. Me ha dicho vale, vamos a preguntar al abogado eso. A la hora de recoger le digo eso has dicho que esta tarde vamos al abogado y me dice vamos y gracias a Dios que salió todo bien (ZA_01).

Albuñol es el municipio estudiado donde hemos encontrado una mayor concentración de marroquíes que proceden de los mismos municipios, concretamente de algunas aldeas de la provincia de Larache. Khaled (AL_02) llegó a Albuñol en 2000 con una oferta de trabajo que un hermano emigrado anteriormente tramitó con su empleador. Su relato también muestra el importante papel de la componente familiar de la cadena migratoria: "Mi hermano me trajo aquí con un contrato. Aunque sea desde Marruecos, lo esencial, era conseguirle gente (se refiere al patrón español). Que cuando vinieran aquí, les da su contrato firmado, les firma un contrato. Le dice a mi hermano, tráeme a 3 o 4, y él se los busca". Al cabo de tres años reagrupó a su esposa e hijos. Esta red familiar no siempre funcionó con contratos de trabajo que los familiares emigrados gestionaban con sus empleadores o con visados de reagrupación. Según se desprende de los testimonios recogidos, 
la posibilidad de un contrato no era frecuente en los primeros años: "En ese tiempo tenían miedo los dueños, antes los patrones no podían, tenían miedo de hacer un contrato a una persona... Tú pedías un contrato, no te lo daban... porque como no había tanta inspección..." (AL_03). Cabe pensar que este "miedo" al que hace alusión el entrevistado se refiere a que los agricultores preferían contratar a persona que conocieran o de la que al menos tuvieran referencia. Pero, por otra parte, además de que evidentemente los costes eran menores, el hecho de no contar con un contrato hacía más factible reemplazar a estos trabajadores en caso de que no respondieran a las expectativas.

Vemos que el entrevistado de la primera cita hace hincapié en el hecho de que tuvo que insistir para que el empleador le facilitara un contrato de trabajo para el hermano en Marruecos. Llega un momento en que la cadena adquiere un carácter "mercantil": la compra de contratos de trabajo, por sumas realmente importantes, en el marco de la agricultura intensiva. En esta relación intervienen varios actores: el empresario español, el inmigrado marroquí que ejerce de intermediario y el destinatario del contrato, es decir, el candidato potencial a la emigración.

Aunque menos numerosos, también hemos encontrado casos de jóvenes marroquíes que han montado su propio negocio en España y han procedido a realizar un contrato en origen destinado a uno de los hermanos. Said, originario de Tarudant, regenta un comercio en Granada y se dedica además a la importación de mercancías:

Yo espero ver si contrato a mi hermano, él todavía está estudiando, el menor, a ver si puede venir a España para que me ayude y para que yo pueda volver a estudiar. Porque yo tengo ganas de terminar mi carrera y continuar con mis negocios, no con la carrera. Yo la carrera yo quiero tenerla nada más [...] Pues, yo le cuento..., mi padre no quiere. Pero yo le he dicho para que él venga, para dejarlo en esta tienda. Yo voy a hacer otra nave. Una nave en el futuro. Una nave la voy a tener, porque yo el almacén que tengo en el centro, es muy pequeño (GR_05).

De este último testimonio hay un dato interesante que señalar. De origen soussi, como hemos indicado, parece estar emulando las estrategias empresariales de algunos de sus compatriotas emigrados a Francia en los años 60 y 70, entre quienes era frecuente contratar a familiares durante determinados meses con el fin de mantener el establecimiento todo el año abierto con personal de confianza y, de este modo, realizar los desplazamientos comerciales con mayor tranquilidad. Una estrategia bastante similar a la de 
nuestro joven entrevistado, que afirmó durante nuestro encuentro tener como proyecto extender su negocio de importación y retomar los estudios.

\section{A modo de conclusión}

Los resultados de nuestro estudio nos revelan que, efectivamente, la familia juega un papel esencial en los procesos de partida de los jóvenes marroquíes emigrados a España: a la hora de tomar la decisión, como arrastre e incluso como soporte para sufragar los gastos. Ahora bien, lo que más llama la atención es que, para muchos de estos jóvenes, se trata de una cadena de ayuda entre hermanos que comparten un mismo descontento generacional. Hemos visto que es significativa la probabilidad de que una vez que un joven se lance al periplo migratorio lo hagan posteriormente, en distintos momentos, no un hermano más, sino varios e incluso todos. No son extraños los casos en los que el proyecto migratorio se inicia al margen de los padres, con absoluto desconocimiento.

En la mayoría de los testimonios encontramos las dificultades para incorporarse en la vida laboral de manera satisfactoria o acorde con las expectativas como uno de los detonantes principales para emigrar. La falta de expectativas es casi generalizada, aunque de distinta naturaleza. Jóvenes del medio rural que quieren escapar de un futuro prácticamente programado similar al de sus progenitores. Jóvenes del medio urbano con estudios que persiguen prolongarlos en el extranjero o encontrar mejores oportunidades en el mercado laboral. La alta migraciones de hermanos dentro de una misma familia nos está desvelando cómo ha ido calando una cultura de las migraciones entre las capas más jóvenes de la sociedad marroquí como mecanismo de escape ante ese descontento generacional, en el que si bien la familia continua constituyendo una institución de capital importancia en la sociedad marroquí, también es cierto que estos jóvenes quieren una mayor autonomía e independencia. En definitiva, aunque las redes sociales migratorias son muy vastas y complejas y no se circunscriben estrictamente al círculo familiar más próximo, no hay margen para la duda de que la familia constituye una de las piezas clave en su constitución y posterior desarrollo.

\section{Bibliografía}

BERRIANE, Mohamed. Los focos migratorios marroquíes y la emigración hacia España. In LOPEZ GARCIA, Bernabé; BERRIANE, Mohamed (dir.). Atlas de la inmigración marroquí en España. Madrid: UAM Ediciones, 2004, p. 128-130.

CACHÓN, Lorenzo. La España inmigrante: marco discriminatorio, mercado de trabajo y políticas de integración. Barcelona: Anthropos, 2009. 
EL HARRAS, Mokhtar. Marruecos: la diversificación de las estructuras y de las relaciones familiares. In LOPEZ GARCIA, Bernabé; BERRIANE, Mohamed (dir.). Atlas de la inmigración marroquí en España. Madrid: UAM Ediciones, 2004, p. 34-36.

FARGUES, Philippe. La femme dans les pays arabes: vers une remise en cause du système patriarcal? Populations et sociétés, n. 387, 2003, p. 1-4.

FARGUES, Philippe; LE BRAS, Hervé. Migrants et migrations dans le bassin de la Méditerranée. Les Notes IPEMED, Études et analyses, n. 1, 2009.

GONIN, Patrick; KOTLOK, Nathalie; LIMA, Stephanie. Entre réseaux et territoires, des mobilisations multiséculaires pour le développement. Réseaux migratoires et communes rurales dans la région de Kayes, Mali. Espaces, populations, sociétés, n. 2011-2, 2011, p. 265-278.

GOZDIAK, Elzbieta; BUMP, Micah. Poultry, Apples, and New Immigrants in the Rural Communities of the Shenandoah Valley: An Ethnographic Case Study. International Migration, v. 42, n. 1, 2004, p. 149-164.

IZCARA PALACIOS, Simón Pedro. Redes migratorias versus demanda laboral: los elementos que moldean los procesos migratorios. Convergencia, Revista de Ciencias Sociales, v. 18, n. 57, 2011, p. 39-59.

KARAM, Azza. Los jóvenes en el Mediterráneo: retos y oportunidades culturales de cambio. Quaderns de la Mediterrània, n. 11, 2009, p. 193-200.

KLEY, Stefanie. Explaining migration as a process of cumulative causation in the life of course. Universidad de Bremen, Laboratorio Migremus, 2009. Diponível em: $<$ http://www.migremus.uni-bremen.de/images/stories/workingpapers/kleywp. pdf>. Acceso en: 15.01.2014.

LAZAAR, Mohamed. L'immigration marocaine en Espagne. Hommes et Migrations, n. 1243, 2003, p. 83-93.

MASSEY, Douglas S. The ethnosurvey in theory and practice. International Migration Review, v. 21, n. 4, 1987, p. 1498-1522.

MASSEY, Douglas S.; GOLDRING, Luin; DURAND, Jorge. Continuities in transnational migration: an analysis of nineteen mexican communities. American Journal of Sociology, v. 99, n. 6, 1994, p. 1492-1533.

MAYA, Isidro. La formación de comunidades de inmigrantes: desplazamiento en cadena y contexto de recepción. Aruacaria. Revista Iberoamericana de Filosofía, Política y Humanidades, n. 12, 2004, p. 83-91.

PEDONE, Claudia. Diversificación de las cadenas migratorias ecuatorianas hacia el mercado de trabajo agrícola de Murcia, España. In PEDREÑO CÁNOVAS, Manuel; HERNÁNDEZ PEDREÑO, Manuel (eds.). La Condición Inmigrante. Exploraciones e investigaciones desde la Región de Murcia. Murcia: Universidad de Murcia, 2005, p. 255-271.

PÉREZ MONTEROSAS, Mario. Las redes sociales en la migración emergente de Veracruz a los Estados Unidos. Migraciones Internacionales, v. 2, n. 1, 2003, p. 136-169. 
RACHICK, Hassan. Jeunesse et changement social. In 50 ans de développment humain au Maroc. Universidad de Al Akhawayn, 2005, p. 191-216. Disponible en: <http:// conseilsjeunesse.ma/wp-content/uploads/2013/03/Jeunesse-changement-social. pdf > . Acceso en: 10.12.2013.

\section{Abstract}

\section{Family strategies of young Moroccan migrants to Andalusia (Spain) in recent years}

During the last decade Moroccans have been the population outside of the European Union with the largest influx of immigrants to Spain. It is a young and relatively well-educated population hailing from rural and urban areas. Their reasons for emigrating reveal a fairly widespread juvenile discontent that may vary depending on their social profile. In this article we analyze the role of the family at the time of making the decision to migrate, how many family members are involved, and what strategies they have taken. The results of our study reveal the extent to which family plays an essential role in the migration of young Moroccans to Spain. For many of the young migrants, a chain of aid exists between siblings who share the same generational discontent. This article studies the first stages of the migratory process and additionally presents some changes that have taken place in the Moroccan youth in recent years.

Keywords: immigration, family, Morocco, Spain, youth discontent.

Recebido para publicación en 20/02/2014.

Aceptado para publicación en 21/05/2014. Received for publication in February, 20 2014.

Accepted for publication in May, 21 $1^{\text {th }}, 2014$. 